\title{
GENERALIZED CELLS IN GENERALIZED MANIFOLDS
}

\author{
KYUNG WHAN KWUN AND FRANK RAYMOND ${ }^{1}$
}

1. Introduction. The well-known Schoenflies extension theorem asserts that if $J$ is a simple closed curve in a 2 -sphere then the closure of each of the two complementary domains is a disk with $J$ as boundary. That the analogous theorem in a higher dimensional sphere is false has been shown by numerous counter-examples such as $[1 ; 2]$. There is, however, a generalization along a slightly different line. R. L. Wilder shows that an analogy holds at the level of sphere-like closed generalized manifolds (abbreviation: $\mathrm{gcm}$ ) and generalized closed cells [7, Theorem 9.1, p. 312]. He further shows that if $A$ and $B$ are two generalized closed $n$-cells whose boundaries are homeomorphic, then the space obtained by putting $A$ and $B$ together along their boundaries is a sphere-like $n$-gcm. This is an obvious generalization of the case of the union of two disks along their boundaries. In this note, we demonstrate further similarities between ordinary cells and generalized cells. For the definition of the generalized cell, the reader is referred to Definition 7.10, p. 287 of [7].

For the definition of $n$-gm the reader is referred to Definition 1 , p. 244 of [7] except that we shall require that the local cobetti numbers $p^{r}(x ; X)=0, r \neq n$ and that only the cohomology dimension (with respect to the defining field), be finite instead of an explicit covering $n$-dimensional assumption. We lose nothing from the theory of $n$-gm's with this weaker assumption. For the definition (first given by White [6]) of $n$-gm with boundary the reader is referred to [4]. Again we shall make the change in the dimension assumption. By a (locally) orientable $n$-gm, $M=X \cup B$, with orientable boundary $B$ we mean that $M$ is an $n$-gm with boundary $B$, such that $X$ is a locally orientable $n$-gm, each component $B_{i}$ of $B$ is an orientable $(n-1)$-gm with the property that $B_{i}$ is contained in some orientable neighborhood $U_{i}$ of $M, U_{i} \cap U_{j}=\phi$, for $i \neq j$.

In this note, the mapping theorem of Wilder [8] easily yields:

Theorem 1. Let $M=X \cup B$ be a (locally) orientable n-gm with orientable boundary $B$. Suppose each component $B_{i}$ of $B$ is a spherelike $(n-1)-\mathrm{gcm}$. Then the space $M^{*}$ obtained by identifying each $B_{i}$ to a point $x_{i}$ is a (locally) orientable $n$-gm.

Proof. First, if $C=S \vee p$ is a cone over a space $S$ with vertex $p$,

Received by the editors April 27, 1959.

1 The second-named author holds a National Science Foundation Fellowship. The first-named author is now at Seoul National University, Seoul, Korea. 
it is easy to see that the $i$ th local betti number of $C$ at $p$ is the $(i-1)$ th betti number of $S$. Furthermore, as $C-p$ is a product of $S$ with a ray it follows that $C-p$ is an orientable $n$-gm with orientable boundary $S$, if $S$ is an orientable $(n-1)$-gm, [5]. If $S$ is a sphere-like orientable $(n-1)-\mathrm{gcm}$, then $C$ is an orientable $n$-gm with orientable boundary $S$ and hence, in this case, is a generalized closed $n$-cell.

For each $B_{i}$ of $M$ choose an ideal point $p_{i}$ and form the cone $B_{i} \vee p_{i}$ over $B_{i}$. The space $M^{\prime}=M \cup\left(\cup_{i}\left(B_{i} \bigvee p_{i}\right)\right)$ is an $n$-gm without boundary according to [4]. The $n$-gm $M^{\prime}$ is orientable or locally orientable depending on whether $X$ is locally orientable or orientable. Let $f: M \rightarrow M^{*}$ by identifying each cone $B_{i} \bigvee p_{i}$ to a point $x_{i}$. The space $M^{*}$ is clearly the space $M^{*}$ of the statement of the theorem obtained by identifying each $B_{i}$ of $M$ to a point $x_{i}$. As the inverse image $f^{-1}\left(m^{*}\right)$ of each point $m^{*} \in M^{*}$ is either a point of $X$ or a generalized closed $n$-cell and hence acyclic, it follows from [8] that $M^{*}$ is a (locally) orientable $n$-gm of the same homology type as $M^{\prime}$.

Remark 1. If $M$ and $X$ are connected, $M$ compact then,

$$
\begin{gathered}
H^{n}\left(M^{*}\right) \approx H_{c}^{n}(X), H^{n-1}(M) \oplus H_{c}^{n}(X) \approx H^{n-1}\left(M^{*}\right) \oplus \sum_{i} H^{n-1}\left(B_{i}\right), \\
H^{p}(M) \approx H^{p}\left(M^{*}\right), \quad p \neq n, n-1,
\end{gathered}
$$

follows immediately from the Mayer-Vietoris sequence for Cech cohomology. ( $H_{c}^{*}$ denotes cohomology with compact supports.) In particular, if $M$ is a generalized closed $n$-cell we have:

COROLLARY 1. The space obtained from a generalized closed $n$-cell by identifying the points of the boundary is a sphere-like $n-\mathrm{gcm}$.

The following seems interesting even for the case in which $C$ is the closure of the interior of an $(n-1)$-sphere imbedded in $E^{n}$.

COROLla Ry 2. Let $C$ be a generalized closed $n$-cell with $K$ as boundary and $C^{\prime}$ be a generalized closed $(n-1)$-cell imbedded in $C$ with $K^{\prime}$ as boundary such that $K^{\prime}=K \cap C^{\prime}$. Then $C^{\prime}$ separates $C$ into 2 domains $D_{1}$ and $D_{2}$ of which $C^{\prime}$ is the common boundary in $C$ and the sets $\bar{D}_{i}$ are generalized closed $n$-cells.

Proof. Adjoin a cone $K \bigvee p$ to $K$ and let $f: C \cup(K \vee p) \rightarrow C^{*}$ be the quotient map of Theorem 1. On $\left(C-C^{\prime}\right)-K$ the map $f$ is a 1-1 local homeomorphism and therefore a homeomorphism. By applying Corollary 1 to $C$ and $C^{\prime}$ simultaneously, we find that $f\left(\left(C-C^{\prime}\right)-K\right)$ is the complement in the $n$-gcm $C^{*}$ of the $(n-1)-\mathrm{gcm} f\left(C^{\prime}\right)$ and has two components. Since $f$ is a homeomorphism on $\left(C-C^{\prime}\right)-K$, 
$\left(C-C^{\prime}\right)-K$ also has two components, say, $D_{1}$ and $D_{2}$. Let $K_{1}$ and $K_{2}$ be two generalized closed $(n-1)$-cells in $K$ such that $K_{1} \cup K_{2}=K$ and $K_{1} \cap K_{2}=K^{\prime}[7$, Theorem 9.1, p. 312]. The generalized closed $(n-2)$-cell $K^{\prime}$ is the common boundary of $K_{1}$ and $K_{2}$ in $K$. The sets $K_{i} \cup C^{\prime}$ are sphere-like $(n-1)$-gcms [7, Theorem 9.2, p. 312] meeting $C$ at subsets of its boundary. Hence, there is a complementary domain $P_{i}$ of $K_{i} \cup C^{\prime}$ in $C \cup(K \vee p)$ meeting no point of $K \bigvee p$. Clearly, $P_{1} \cap P_{2}=\phi$. Furthermore, any connected set in $\left(C-C^{\prime}\right)-K$ is either in one of $P_{i}$ or it does not meet $P_{1} \cup P_{2}$ at all. But $P_{1} \cup P_{2} \subset D_{1} \cup D_{2}$. Then it follows that $D_{1}$ is identical with one of $P_{i}$ and $D_{2}$ with the other. Corollary 2 now follows from [7, Theorem 9.1, p. 312].

In the proof of Theorem 1 we saw that if we formed a cone over a sphere-like orientable $(n-1)$-gcm we obtained a generalized $n$-cell. A type of converse is the following:

Theorem 2. Let $X$ be a locally orientable $n$-gm. Suppose for some $x \in X$, there exists an open set $O$, containing $x$, with compact closure $\bar{O}$ and boundary $O^{\prime}$ and a homeomorphism $h: \bar{O} \rightarrow B \vee p$, a cone, such that $h\left(O^{\prime}\right)=B$ and $h(x)=p$, then $\bar{O}$ is a generalized $n$-cell.

Proof. As $O-x$ is homeomorphic to $O^{\prime} \times E^{1}$, where $E^{1}$ is the open unit interval, it follows from [5] that $O^{\prime}$ is a locally orientable $(n-1)$ $\mathrm{gcm}$. (The paracompactness assumption of [5] is no longer necessary.) As $x$ corresponds to the vertex of the cone and as the $i$ th local betti number of $X$ at $x$ is the same as the $(i-1)$ th betti number of $O^{\prime}$, it follows that $O^{\prime}$ must have sphere-like homology. That $O^{\prime}$ is connected follows from the fact that the oth augmented betti number must be zero. Thus $O^{\prime}$ is an orientable sphere-like $(n-1)-\mathrm{gcm}$ and consequently $\bar{O}$ is a generalized $n$-cell as it is acyclic.

As is well known, any combinatorial 3-manifold is a locally Euclidean 3-manifold. Applying Theorem 2 we establish an analogous criterion for 3-gms:

Corollary 3. A necessary and sufficient condition that a separable metric space $X$ be a classical 3-manifold is that $X$ be a 3-gm, and for each $x \in X$ there exists a compact neighborhood such that the neighborhood is homeomorphic to a cone over the boundary of the neighborhood.

Proof. The necessity is obvious. From Theorem 2 we have seen that the compact neighborhood is a cone over an orientable spherelike $2-\mathrm{gcm}$. (That $X$ be locally orientable is not needed since the boundary of the cone must be a 2-gm, and consequently, in this case, must be an orientable $2-\mathrm{gcm}$.) Of course, here $x$ need not correspond to the vertex of the cone. The only separable metric orientable sphere- 
like $2-\mathrm{gcm}$ is the 2 -sphere, and thus, the corollary follows.

The suspension of a Poincaré space shows that the corollary can not be extended past dimension 3 .

REMARK 2. The results of this note are valid for generalized manifolds defined over the integers, $Z$, as well as over a field. The definition of an $n$-gm, $X$, over $Z$ is analogous to that over a field. When using integer coefficients, the condition that the local cobetti number, $p^{n}(x ; X)=k$, means that given a neighborhood $U$ of $x$ we can find neighborhoods $V$ and $W$ of $x$, so that, if $x \in W^{\prime} \subset W \subset V \subset U$, then the image of $H_{c}^{n}\left(W^{\prime} ; Z\right)$ in $H_{c}^{n}(V, Z)$ under the inclusion is the same as the image of $H_{c}^{n}(W ; Z)$ in $H_{c}^{n}(V ; Z)$ and is free of rank $k$. Orientability of $X$, over $Z$, is just the requirement that $j^{*}: H_{c}^{n}(V ; Z) \rightarrow H_{c}^{n}(U ; Z)$ is an isomorphism onto for all connected open sets $V$ and $U$ with compact closures, $V \subset U$.

The universal coefficient theorem for Cech cohomology with compact supports easily implies that $X$ is a (orientable, or locally orientable) $n$-gm over any field if $X$ is a (orientable, or locally orientable) $n$-gm over $Z$. On the other hand, if $X$ is an orientable $n$-gm over every field, ( $n$ depending upon the field), it is unknown whether $X$ is an orientable $n$-gm over $Z$. However we can show:

Proposition. If $X$ is clc over $Z, \operatorname{dim}_{Z} X<\infty$, and $X$ is an orientable $n$-gm over $Q$, the rationals, and $Z_{p}$, the integers modulo a prime $p$, for all primes $p$, then $X$ is an orientable $n$-gm over $Z$.

We remark that the $n$ of the hypothesis is fixed. Nearly each step of the proof uses the universal coefficient theorem and the fact that since $X$ is clc over $Z, \operatorname{dim}_{Z} X<\infty$, then given $V$, an open set whose closure, compact, is contained in an open set, $U$, the image of $H_{c}^{*}(V)$ in $H_{c}^{*}(U)$ is finitely generated [3]. One may fill in the details of the outline of proof we shall give by constructing the appropriate diagrams and using the facts we have just mentioned.

(i) $\operatorname{dim}_{z} X=n$.

(ii) If $V$ is open, connected, $\bar{V}$ compact, $\subset U$, open, then $\left(H_{c}^{n}(V ; Z) \subset H_{c}^{n}(U ; Z)\right) \approx Z$, where $\left(H_{c}^{n}(V ; Z) \subset H_{c}^{n}(U ; Z)\right)$ means the image of $H_{c}^{n}(V ; Z)$ in $H_{c}^{n}(U ; Z)$ under the map $j^{*}$ induced by the inclusion $j: V \subset U$.

(iii) If $V \subset \bar{V} \subset U \subset \bar{U} \subset W, \bar{U}$ compact, $U$ connected, then $\left(H_{c}^{n}((U-V) ; Z) \subset H_{c}^{n}((W-V) ; Z)\right)=0$.

(iv)If $V \subset \bar{V} \subset U \subset \bar{U} \subset W$ then $\left(H_{c}^{n}(V ; Z) \subset H_{c}^{n}(W ; Z)\right)$ $=\left(H_{c}^{n}(U ; Z) \subset H_{c}^{n}(W ; Z)\right) \approx Z, U$ and $V$ connected, $\bar{U}$ compact.

(v) We may as well assume $X$ connected. Let $V, W$ be two open 
connected sets with compact closures; then $\left(H_{c}^{n}(V ; Z) \subset H_{c}^{n}(X ; Z)\right)$ $=\left(H_{c}^{n}(W ; Z) \subset H_{c}^{n}(X ; Z)\right) \approx Z$.

(vi) The local cobetti numbers $p^{r}(x ; X)=0, r \neq n n$. Thus, $X$ is an orientable $n$-gm over $Z$.

Using the above proposition one can extend Wilder's mapping theorem [8], to generalized manifolds over the integers. Another type of extension one can obtain is that the Cartesian product of two (locally) orientable generalized manifolds over $Z$ is a locally orientable generalized manifold over $Z$, and conversely, if a (locally) orientable generalized manifold over $Z$ is a product of two spaces then both factors must be (locally) orientable generalized manifolds, over $Z$.

Hence the theorems and corollaries of this note can be extended to generalized manifolds and generalized cells defined over $Z$.

\section{BIBLIOGRA PHY}

1. J. Alexander, An example of a simply connected surface bounding a region which is not simply connected, Proc. Nat. Acad. Sci. U.S.A. vol. 10 (1924) pp. 8-10.

2. E. Artin and R. Fox, Some wild cells and spheres in three dimensional space, Ann. of Math. vol. 49 (1948) pp. 979-990.

3. A. Borel, The Poincare duality in generalized manifolds, Michigan Math. J. vol. 4 (1957) pp. 227-241.

4. T. Brahana, $A$ theorem about local betti groups, Michigan Math. J. vol. 4 (1957) pp. 33-37.

5. F. Raymond, On factorization of generalized manifolds, Notices Amer. Math. Soc. vol. 5 (1958) p. 298.

6. P. White, On the union of two generalized manifolds, Ann. Scuola Norm. Sup. Pisa vol. 4 (1950) pp. 231-243.

7. R. Wilder, Topology of manifolds, Amer. Math. Soc. Colloquium Publications, vol. 32, 1949, 402 pp.

8. —, Montone mappings of manifolds II, Michigan Math. J. vol. 5 (1958) pp. $19-25$.

TUlane University AND

The Institute for Advanced Study 